\title{
Livros eletrônicos da área médica
}

\author{
E-books for the medical profession
}

\author{
Sonia Pedrozo Gomes ${ }^{1}$
}

\section{RESUMO}

O trabalho indica alguns livros eletrônicos gratuitos disponíveis na Internet e destinados à área médica.

Palavras-chaves: Livro eletrônico. Apontadores para endereços na Internet.

\section{ABSTRACT}

This paper indicates some e-books (electronic books) that are available on the Internet for free access and destined for the medical profession. Key-words: Electronic book. Links for Internet addresses.

$\mathrm{Na}$ Internet podemos encontrar uma série de recursos para se estar bem informados. Segundo atesta Lopes " "as publicações eletrônicas disponibilizadas na Web constituem um dos temas de maior repercussão dentro da comunidade científica, permitindo o acesso, produção e disseminação de informação em larga escala, por um único indivíduo ou por organizações, revolucionando toda a estrutura desta produção, disseminação e acesso que estava em vigor antes do advento da Internet".

Uma boa alternativa é o acesso aos conteúdos de domínio público sem direitos autorais ou com distribuição liberada para fins não-comerciais.

Em $2003^{1}$, foi indicada vasta biblioteca de livros eletrônicos, com os seus respectivos endereços na Internet, escolhidos de maneira que auxiliasse a qualquer pessoa que trabalhe ou estude na área de saúde. Agora, aqui estão mais oitenta e oito livros eletrônicos selecionados conforme os mesmos critérios adotados anteriormente.

Os formatos dos livros eletrônicos variam desde HTML até DOC e TXT; porém, o mais comum de ser encontrados são os PDF (Portable Document Format), que necessita do programa Acrobat Reader para a sua leitura na própria tela do computador e pode ser adquirido gratuitamente no site http://www.adobe.com.br .

A praticidade de armazenar os títulos depois, de ter feito 0 download, em disco rígido ou, ainda, em CD e/ou DVD, mais a facilidade de realizar buscas por palavras ou trechos em todo seu conteúdo, faz com que seu uso seja recomendado.

Com relação ao artigo de 2003, alguns endereços relacionados aos livros eletrônicos, publicados pela FUNASA, foram desativados, outros foram redirecionados. Como conseguimos recuperar alguns desses endereços, estamos listando-os no final do artigo.

\section{AIDS}

AIDS - leia antes de escrever. Guia prático para profissionais de comunicação. Brasília, Ministério da Saúde, Secretaria de Vigilância em Saúde, Programa Nacional de DST/Aids, 2003. (80KB)

http://www.aids.gov.br/services/DocumentManagement/FileDownload.EZTSvc.asp?DocumentID=\{09F7D6AB-96E9-46298CE9-0D648A28D990 $\} \&$ ServiceInstUID $=\{$ B8EF5DAF-23AE-4891-AD36-1903553A3174 $\}$

\author{
http://www.aids.gov.br/leia/leia.htm
}

http://www.aids.gov.br/data/documents/storedDocuments/\%7BB8EF5DAF-23AE-4891-AD36-1903553A3174\%7D/ \%7BFA4AB810-A15F-4C80-824C-E6E4A1B91605\%7D/leia.pdf

\footnotetext{
1. Instituto de Medicina Tropical de São Paulo, Universidade de São Paulo, São Paulo, SP.

Endereço para correspondência: Sonia Pedrozo Gomes. Biblioteca do Instituto de Medicina Tropical de São Paulo/USP/Biblioteca Setorial da FMUSP. Av. Dr. Enéas de Carvalho Aguiar 470, 05403-000 São Paulo, SP.

Tel: 5511 3061-7003.

e-mail: spgomes@usp.br

Recebido para publicação em 22/2/2007

Aceito em 16/4/2007
} 
Critérios de definição de casos de aids em adultos e crianças. Brasília, Ministério da Saúde,Secretaria de Vigilância em Saúde,Programa Nacional de DST e Aids, 2003.

58p. (Série Manuais no. 60) (802KB)

http://www.giv.org.br/publicacoes/criterios.pdf

Programa Estratégico de Ações Afirmativas: população negra e Aids. Brasília, Ministério da Saúde, 2005. 19p.

http://bvsms.saude.gov.br/bvs/publicacoes/populacao negra e aids.pdf

\section{ATESTADOS DE ÓBITO}

Declaração de óbito: documento necessário e importante. Brasília, Ministério da Saúde, Conselho Federal de Medicina, 2006.40 p. (Série A Normas e Manuais Técnicos) (1.74MB)

ISBN 85-334-1170-7 Ministério da Saúde

ISBN 85-870-7704-X Conselho Federal de Medicina

http://www.portalmedico.org.br/arquivos/cartilha_do_cfm_ms.pdf

\section{ATLAS DE ANATOMIA}

Atlas Anatômico P.R. Mede-mécum Brasil on-line Brasil. Imagens didáticas sobre os principais órgãos e sistemas, os sentidos e o corpo humano. (On line)

http://www.prvademecum.com/PrVademecum Bra/Apendice/bra atlas.asp

\section{BIOLOGIA MOLECULAR}

Housset C, Raisonnier A. Biologie moléculaire. Paris, Faculté de Médecine Pierre et Marie Curie, 2006-2007. 204p. (3.60MB)

http://www.chups.jussieu.fr/polys/biochimie/BMbioch/bmbioch.pdf

http://www.chups.jussieu.fr/polys/biochimie/BMbioch/

\section{BIOSSEGURANÇA}

Biossegurança em laboratórios biomédicos. 2.ed.rev.ampl. Brasília, Ministério da Saúde, 2004. 290p. (Série A Normas e Manuais Técnicos) (1398KB)

ISBN 85-334-0777-7

http://portal.saude.gov.br/portal/arquivos/pdf/livro biossegurança 2005.pdf

Diretrizes gerais para o trabalho em contenção com material biológico. Brasília, Ministério da Saúde, Secretaria de Ciência, Tecnologia e Insumos Estratégicos, 2004. 60p. (Série A Normas e Manuais Técnicos) (333KB)

ISBN 85-334-0793-9

http://dtr2001.saude.gov.br/editora/produtos/livros/pdf/04_0408_M.pdf

Procedimentos para a manipulação de microorganismos patogênicos e/ou recombinantes na FIOCRUZ. Rio de Janeiro, Comissão Técnica de Biossegurança da FIOCRUZ,

CTBio FIOCRUZ, 2005. 221p. (4274KB)

http://www.biossegurancahospitalar.com.br/files/LivroProcedManipMicroPato.pdf

Dutra SMD, Rubin MRB. Manual de orientações para coleta, preparo e transporte de material biológico - LACEN/SC. Santa Catarina, Secretaria de Estado da Saúde, Laboratório Central de Saúde Pública de Santa Catarina, 2006. 92p.

http://www.saude.sc.gov.br/hospitais/lacen/Manual Coleta 2006.pdf 


\section{DAPHNIA}

Ebert D. Ecology, epidemiology, and evolution of parasitism in Daphnia. Bethesda, National Library of Medicine, National Center for Biotechnology Information, 2005. 110p. (17.8MB)

ISBN 1-932811-06-0

http://www.ncbi.nlm.nih.gov/books/bookres.fcgi/daph/screenA4.pdf

http://www.ncbi.nlm.nih.gov/books/bv.fcgi?rid=daph.TOC\&depth=2

\section{DENGUE}

Dengue diagnóstico manejo clínico. Brasília, Ministério da Saúde, Secretaria de Vigilância em Saúde, Diretoria Técnica de Gestão, 2005. 24p. (Série A Normas e Manuais Técnicos) (423KB) (Apesar do link indicar 2006 o livro é de 2005)

ISBN 5-334-1020-44

http://portal.saude.gov.br/portal/arquivos/pdf/dengue manejo clinico 2006.pdf

Dengue : roteiro para capacitação de profissionais médicos no diagnóstico e tratamento. Manual do aluno. 2.ed. Brasília, Ministério da Saúde, Secretaria de Vigilância em Saúde, Diretoria Técnica de Gestão, 2005. 72 p. (Série A Normas e Manuais Técnicos) (1.04MB) ISBN 85-334-1026-3

http://portal.saude.gov.br/portal/arquivos/pdf/apostila dengue aluno.pdf

Dengue : roteiro para capacitação de profissionais médicos no diagnóstico e tratamento. manual do monitor. 2.ed. Brasília, Ministério da Saúde, Secretaria de Vigilância em Saúde, Diretoria Técnica de Gestão, 2005. 72 p. (Série A Normas e Manuais Técnicos) (423KB)

ISBN 85-334-1041-7

http://portal.saude.gov.br/portal/arquivos/pdf/apostila dengue monitor.pdf

Guia básico de dengue: para órgãos públicos e privados, comércio, pequenas e grandes empresas. São Paulo, Secretaria da Saúde do Estado de São Paulo, Superintendência de Controle de Endemias, Diretoria de Combate a Vetores, 2002. 28p. (418KB)

http://www.sucen.sp.gov.br/docs_tec/dengue\%20.pdf

\section{DIREITO SANITÁRIO}

Manual de direito sanitário com enfoque na vigilância em saúde. Brasília, Ministério da Saúde, Secretaria de Vigilância em Saúde, 2006. 132 p. (Série E. Legislação de Saúde) (1010KB)

ISBN 5-334-1259-22

http://portal.saude.gov.br/portal/arquivos/pdf/manual direito sanitario.pdf

Questões atuais de direito sanitário. Brasília, Ministério da Saúde, Editora do Ministério da Saúde, 2006. 202 p. (Série E Legislação de Saúde) (1456KB)

ISBN 85-334-1064-6

http://dtr2001.saude.gov.br/editora/produtos/livros/pdf/06 0860 M.pdf

http://dtr2001.saude.gov.br/editora/produtos/livros/zip/06 0860 M.zip

\section{DIRETRIZES MÉDICAS}

Projeto diretrizes. São Paulo, Associação Médica Brasileira, Brasília, Conselho Federal de Medicina, 2007. 4v. (on-line)

http://www.projetodiretrizes.org.br/ 


\section{DOENÇAS FALCIFORMES}

Manual de condutas básicas na doença falciforme. Brasília, Ministério da Saúde, Secretaria de Atenção à Saúde, Departamento de Atenção Especializada, Editora do Ministério da Saúde, 2006. 56 p. (Série A Normas e Manuais Técnicos) (427KB)

ISBN 85-334-1090-5

http://dtr2001.saude.gov.br/editora/produtos/livros/pdf/06_0241_M.pdf

http://dtr2001.saude.gov.br/editora/produtos/livros/zip/06 0241 M.zip

\section{DOENÇAS INFECCIOSAS}

Blue book - Guidelines for the control of infectious diseases. Revised edition 2005.

Melbourne, Communicable Diseases Section, Public Health Group, Victorian Department of Human Services, 2005. 270p. (1MB)

http://www.health.vic.gov.au/ideas/downloads/bluebook.pdf

http://www.health.vic.gov.au/ideas/bluebook/index.htm

www.health.vic.gov.au/ideas/bluebook

Doenças infecciosas e parasitárias. Guia de bolso. 4.ed.ampl. Brasília, Ministério da Saúde: Fundação Nacional da Saúde, 2004. 334 p. (3756KB)

http://portal.saude.gov.br/portal/arquivos/pdf/guia bolso 4ed.pdf

Doenças infecciosas e parasitárias: guia de bolso. 5.ed.ampl. Brasília, Secretaria de Vigilância em Saúde, Ministério da Saúde, 2005. 320 p. (Série B Textos Básicos de Saúde) (3198KB)

ISBN 85-334-1048-4

http://bvsms.saude.gov.br/bvs/publicacoes/guia_bolso_5ed2.pdf

Doenças infecciosas e parasitárias: guia de bolso. 6.ed.rev. Brasília, Ministério da Saúde, Secretaria de Vigilância em Saúde, 2006. 320p. (Série B Textos Básicos de Saúde)

ISBN 85-334-1222-3

http://portal.saude.gov.br/portal/arquivos/pdf/guia bolso 6ed.pdf

\section{DOENÇAS OCUPACIONAIS}

Dias EC (org). Doenças relacionadas ao trabalho: manual de procedimentos para os serviços de saúde. Brasília, Ministério da Saúde do Brasil, Organização Pan-Americana da Saúde no Brasil, 2001. 580 p. (Série A Normas e Manuais Técnicos; n.114) (5972KB)

ISBN 85-334-0353-4

http://www.portalmedico.org.br/download/arquivos/saudedotrabalhador.zip

\section{DOENÇAS SEXUALMENTE TRASMISSÍVEIS}

Aprendendo sobre aids e doenças sexualmente transmissíveis. Livro da família. 3.ed. Ministério da Saúde, 2001. 84p. (9MB)

ISBN : 85-334-0178-7

http://www.aids.gov.br/services/DocumentManagement/FileDownload.EZTSvc.asp?DocumentID=\{B6AA55DF-C268-4F6DB870-9F82A569E0DE $\}$ \&ServiceInstUID $=\{$ B8EF5DAF-23AE-4891-AD36-1903553A3174 $\}$

Guia de tratamento clínico da infecção pelo HIV em pediatria. 3.ed. Brasília, Ministério da Saúde, Secretaria de Vigilância em Saúde, Programa Nacional de DST e Aids, 2006. 168p. (iSérie Manuais, No.18) (2.2MB)

http://www.aids.gov.br/services/DocumentManagement/FileDownload.EZTSvc.asp?DocumentID=\{50AF4760-5C32-4C94BE52-CCD14E387A41 $\}$ \&ServiceInstUID $=\{$ B8EF5DAF-23AE-4891-AD36-1903553A3174 $\}$ 
Manual de controle das doenças sexualmente transmissíveis. 4.ed. Brasília, Ministério da Saúde, Secretaria de Vigilância em Saúde, Programa Nacional de DST/Aids, 2006. 140p. (Série Manuais No. 68) (1790KB)

http://www.aids.gov.br/data/documents/storedDocuments/\%7BB8EF5DAF-23AE-4891-AD361903553A3174\%7D/\%7B43F95B4B-CD35-4B62-981A-60A62945E318\%7D/manual dst tratamento.pdf

Manual de bolso das doenças sexualmente transmissíveis. 2.ed. Brasília, Ministério da Saúde, Secretaria de Vigilância em Saúde, Programa Nacional de DST e Aids, 2006. 111p. (Série Manuais No. 24) (1006KB)

http://www.aids.gov.br/data/documents/storedDocuments/\%7BB8EF5DAF-23AE-4891-AD36-1903553A3174\%7D/ \%7BB7B9E37F-599B-4F64-969A-082CC8D95F92\%7D/manual\%20dst_bolso.pdf

Paiva C. Cartilha eu gosto de ser mulher: sobre corpo, saúde e direitos da mulher. Brasília, Ministério da Saúde, Coordenação Nacional de DST/Aids, 2002. (1.81MB)

http://www.aids.gov.br/services/DocumentManagement/FileDownload.EZTSvc.asp?DocumentID=\{9118EFA9-F1B8-495B2E3F-B62E42E089EE $\}$ \&ServiceInstUID $=\{$ B8EF5DAF-23AE-4891-AD36-1903553A3174 $\}$

Paiva M. 0 gatão e seus amigos. De homem pra homem. 0 sexo numa visão masculina. Brasília, Ministério da Saúde, Programa Nacional de DST/Aids, s.d. (1.05MB)

http://www.aids.gov.br/services/DocumentManagement/FileDownload.EZTSvc.asp?DocumentID $=\{$ CEA58415-42CF-4C0DBAFC-728123E9D004 $\} \&$ ServiceInstUID $=\{$ B8EF5DAF-23AE-4891-AD36-1903553A3174 $\}$

\section{EDUCAÇão EM SAÚDE vide SAÚDE PÚBLICA}

\section{GEOGRAFIA MÉDICA}

Atlas Racial Brasileiro 2005. Software de Banco de dados eletrônico que reúne mais de cem indicadores sociais abertos por raça/cor, para o Brasil e, sempre que possível, desagregados por sexo, e para as grandes regiões e as unidades da federação (UFs). Brasília, Programa das Nações Unidas para Desenvolvimento (PNUD), 2005. (É instalado no computador)

http://www.aids.gov.br/services/DocumentManagement/FileDownload.EZTSvc.asp?DocumentID=\{E2006DD8-65C9-42C3-

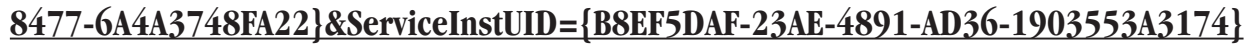

http://www.pnud.org.br/publicacoes/atlas racial/index.php

\section{HANSENÍASE}

Guia para o controle da hanseníase. Brasília, Ministério da Saúde, Secretaria de Políticas de Saúde, 2002. (Versão preliminar) 90 p. (Série Cadernos de Atenção Básica; n. 10) - (Série A. Normas e Manuais Técnicos; n. 111) (1006KB)

ISBN 85-334-0346-1

http://dtr2001.saude.gov.br/editora/produtos/livros/pdf/02 0312 M.pdf

http://dtr2001.saude.gov.br/editora/produtos/livros/zip/02 0312 M.zip

\section{HEPATITES}

A, B,C,D,E de hepatites para comunicadores. Brasilia, Ministério da Saúde, Secretaria de Vigilância em Saúde, Departamento de Vigilância Epidemiológica, 2005. 24 p. (Série F.Comunicação e Educação em Saúde) (343KB)

ISBN 85-334-1012-3

http://portal.saude.gov.br/portal/arquivos/pdf/hepatites abcde.pdf 
Hepatites virais: o Brasil está atento. Brasília, Ministério da Saúde, Secretaria de Vigilância em Saúde, Departamento de Vigilância Epidemiológica da Saúde, 2005. 40 p. (Série Textos Básicos de Saúde) (639KB)

ISBN 85-334-1202-9

http://portal.saude.gov.br/portal/arquivos/pdf/hepatites virais brasil atento.pdf

\section{HEMOVIGILÃNCIA vide TRANSFUSÃo DE SANGUE}

\section{HISTOLOGIA}

Branco DM, Muller RL. Atlas eletrônico de histologia. Porto Alegre, Departamento de Ciências Morfológicas; Departamento de Informática Aplicada; Universidade Federal do Rio Grande do Sul, 1995.

http://www.inf.ufrgs.br/ branco/atlas.zip ((1.76MB)

http://www.danielbranco.com.br/atlasi/atlas.html

http://www.dominiopublico.gov.br/pesquisa/DetalheObraDownload.do?select action=\&co obra=20054\&co midia=2 (3.33MB)

http://www.dominiopublico.gov.br/pesquisa/DetalheObraForm.do?select_action=\&co_obra=20054

HIV

Alimentação e nutrição para pessoas que vivem com HIV e Aids. Brasilia, Ministério da Saúde, Secretaria e Vigilância em Saúde, Secretaria de Atenção à Saúde, Programa Nacional de DST/Aids, 2006. 60p. (Série F. Comunicação e Educação em Saúde) (760Kb)

ISBN 85-3334-1152-99

http://www.aids.gov.br/services/DocumentManagement/FileDownload.EZTSvc.asp?DocumentID $=\{$ EAD3A762-BB53-4E939D1C-C2423ADF132B $\} \&$ ServiceInstUID $=\{$ B8EF5DAF-23AE-4891-AD36-1903553A3174 $\}$

Glosario del VIH/SIDA. 5.ed. Rockville, AIDSinfo, 2006. 196p. (1512KB)

http://aidsinfo.nih.gov/contentfiles/SpanishGlossary_sp.pdf

Glossary of HIV/AIDS. Related terms. Rockville, AIDSinfo, 2005. 174p. (1337KB)

http://aidsinfo.nih.gov/ContentFiles/GlossaryHIV-relatedTerms FifthEdition en.pdf

Hoffman C, Rockstroh JK, Kamps BS (eds) HIV Medicine 2006. 14. ed. Paris, Flying Publisher, 2006. 825p. (5.3MB)

ISBN: 3-924774-50-1

ISBN-13: 978-3-924774-50-9.

http://www.hivmedicine.com/hivmedicine2006.pdf

Manual clínico de alimentação e nutrição na assistência a adultos infectados pelo HIV. Brasília, Ministério da Saúde, Secretaria de Vigilância em Saúde, Programa Nacional de DST/Aids, 2006. 88p. (Coleção DST/Aids Série Manuais, No. 71) (988KB)

ISBN 85-3334-1153-77

http://www.aids.gov.br/services/DocumentManagement/FileDownload.EZTSvc.asp?.DocumentID=\{56D097E4-12DE-429C284C-5E90B48E5DF5 $\}$ \&ServiceInstUID $=\{$ B8EF5DAF-23AE-4891-AD36-1903553A3174 $\}$

Recomendações para profilaxia da transmissão vertical do HIV e terapia anti-retroviral em gestantes. (Versão preliminar) Brasília, Ministério da Saúde, Secretaria de Vigilância em Saúde, Programa Nacional de DST e Aids, 2006. 81p. (Versão preliminar) (347KB)

http://www.aids.gov.br/services/DocumentManagement/FileDownload.EZTSvc.asp?DocumentID=\{723DC082-09A9-4367B51B-438A38E34981 $\} \&$ ServiceInstUID $=\{$ B8EF5DAF-23AE-4891-AD36-1903553A3174 $\}$ 
Recomendações para terapia anti-retroviral em adultos e adolescentes infectados pelo HIV 2004. Brasília, Ministério da Saúde, Secretaria de Vigilância em Saúde, Programa Nacional DST e Aids, 2004. 54p. (969KB)

http://www.giv.org.br/publicacoes/consenso 2004.pdf

\section{HIV - DIREITOS CIVIS}

HIV nos tribunais. 2.ed. Brasília, Ministério da Saúde, Coordenação Nacional de DST e Aids, 1999. 83p. (1007Kb)

http://www.aids.gov.br/services/DocumentManagement/FileDownload.EZTSvc.asp?DocumentID=\{2E76BC65-E31E-4949A3E7-D2C222914077\}\&ServiceInstUID=\{B8EF5DAF-23AE-4891-AD36-1903553A3174\}

\section{HIV - DIREITOS HUMANOS}

Reis T, Harrad D. Projeto Somos. Desenvolvimento organizacional, advocacy e intervenção para ONGs que trabalham com gays e outros HSH. Guia prático. Brasília, Ministério da Saúde, Secretaria de Vigilância em Saúde, Programa Nacional de DST/ Aids, 2005. 141p. (Série Manuais No. 65) (973KB)

http://www.aids.gov.br/services/DocumentManagement/FileDownload.EZTSvc.asp?DocumentID=\{3AE4C7EF-75A4-48D82F21-A8233E4BC9E3 $\}$ \&ServiceInstUID $=\{$ B8EF5DAF-23AE-4891-AD36-1903553A3174 $\}$

\section{HIV-1 - CARGA VIRAL}

Manual de carga viral em HIV-1. São Paulo, Roche Diagnóstica Brasil, Bayer, BioMérieux Brasil, s.d. 231p. (8.3MB)

http://www.aids.gov.br/services/DocumentManagement/FileDownload.EZTSvc.asp?DocumentID=\{2D9FD3C9-0CC1-45982FE4-9AB36F93E10D $\} \& S e r v i c e I n s t U I D=\{A 07528 E 1-7 F B 7-4 C C 7-97 A D-B 7 C B 17 C 9 C A 85\}$

\section{INFLUENZA}

Kamps BS, Hoffman C, Preiser W (eds) Influenza report 2006. Paris, Flying Publisher, 2006. 225p. (2.7MB)

ISBN 3-924774-51-X

http://www.InfluenzaReport.com/InfluenzaReport2006.pdf

\section{LEGISLAÇÃO}

Estatuto da criança e do adolescente. 3.ed. Brasília, Editora do Ministério da Saúde, 2006.

96p. (Série E Legislação de Saúde) (860KB)

ISBN 85-334-1058-1

http://bvsms.saude.gov.br/bvs/publicacoes/lei_8069_06_0117_M.pdf

Estatuto do idoso. 2. ed. rev. Brasilia, Editora do Ministério a Saúde, 2006. 70p. (Série E Legislação de Saúde) (262KB)

ISBN 5-334-1059-XX

http://bvsms.saude.gov.br/bvs/publicacoes/lei_10741_06 0118 M.pdf

Legislação relativa aos sistemas de informações sobre mortalidade (SIM) e sobre nascidos vivos (SINASC). Brasília, Ministério da Saúde, Secretaria de Vigilância em Saúde, Departamento de Análise de Situação de Saúde, 2005. 56p. (409KB)

http://tabnet.datasus.gov.br/tabdata/sinasc/dados/nov/docs/Legisla\%C3\%A7\%C3\%A30\%20PDF.pdf 


\section{LEISHMANIOSE CUTÂNEA}

Atlas de leishmaniose tegumentar americana: diagnósticos clínico e diferencial. Brasília, Editora do Ministério da Saúde, 2006.136 p. (Série Normas e Manuais Técnicos) (11MB)

ISBN 85-3334-0949-44

http://portal.saude.gov.br/portal/arquivos/pdf/atlas lta.pdf

\section{LEISHMANIOSE VISCERAL}

Camargo-Neves VLF (coord). Manual de vigilância e controle da leishmaniose visceral americana do Estado de São. São Paulo, Secretaria de Estado da Saúde, 2006. (11756KB)

ftp://ftp.cve.saude.sp.gov.br/doc tec/zoo/lva06 manual.pdf

http://www.sucen.sp.gov.br/docs_tec/manual\%20lva.pdf

Leishmaniose visceral grave: normas e condutas. Brasília, Ministério da Saúde, Secretaria de Vigilância em Saúde, Departamento de Vigilância Epidemiológica, 2006. 60 p. (Série A Normas e Manuais Técnicos) (478KB)

ISBN 85-3334-0996-66

http://dtr2001.saude.gov.br/editora/produtos/livros/pdf/06 0072 M.pdf

http://dtr2001.saude.gov.br/editora/produtos/livros/zip/06 0072 M.zip

Manual de vigilância e controle da leishmaniose visceral. Brasília, Editora do Ministério da Saúde, 2006. 120 p. (Série A. Normas e Manuais Técnicos) (3800KB)

ISBN 85-334-0742-4

http://portal.saude.gov.br/portal/arquivos/pdf/manual leish visceral2006.pdf

\section{MALÁRIA}

Febre: cuidado, pode ser malária. Brasília, Ministério da Saúde, Secretaria de Vigilância em Saúde, Coordenação-Geral do Programa Nacional de Controle da Malária, 2003. 24 p. (Série A Normas e Manuais Técnicos) (325KB)

ISBN 85-334-0679-7

http://dtr2001.saude.gov.br/editora/produtos/livros/pdf/03 1201 M.pdf

http://dtr2001.saude.gov.br/editora/produtos/livros/zip/03 1201 M.zip

Manual de diagnóstico laboratorial da malária. Brasília, Ministério da Saúde, 2005. 112 p. (Série A.Normas e Manuais Técnicos) $(3812 \mathrm{~KB})$

ISBN 85-334-0974-5

http://portal.saude.gov.br/portal/arquivos/pdf/malaria diag manual final.pdf

MEDICINA

Andrade EO, Gouveia VV, Carneiro MB, Pinheiro AG (coords.). 0 médico e seu trabalho: resultados da região norte e seus estados. Brasília, Conselho Federal de Medicina, 2005. 285p. (1931KB)

ISBN 85-87077-08-2

http://www.portalmedico.org.br/include/biblioteca virtual/medico e seu trabalho/regiao norte/NORTE.pdf

Carneiro MB, Gouveia VV (coords). o médico e o seu trabalho: aspectos metodológicos e resultados do Brasil. Brasília, Conselho Federal de Medicina, 2004. 234 p. (6084KB)

ISBN 85-87077-03-1

http://www.portalmedico.org.br/include/biblioteca virtual/medico e seu trabalho/trabalho.PDF 
Carneiro MB, Gouveia VV, Araújo EJ. 0 médico e seu trabalho: resultados da região sul e seus estados. Brasília, Conselho Federal de Medicina, 2005. 143 p. (3380KB)

http://www.portalmedico.org.br/include/biblioteca virtual/medico e seu trabalho/regiao sul/Livro Sul CFM.PDF

Carneiro MB, Gouveia VV, Chacel PM (coords). 0 médico e seu trabalho: resultados da região centro-oeste e seus estados. Brasilia, Conselho Federal de Medicina, 2005. 208p. (1634KB)

ISBN 85-87077-09-0

http://www.portalmedico.org.br/include/biblioteca virtual/medico e seu trabalho/regiao centro oeste/CENTRO OESTE.PDF

Carneiro MB, Gouveia VV, Pimentel JP. 0 médico e seu trabalho: resultados da região nordeste e seus estados. Brasília, Conselho Federal de Medicina, 2005. 299 p. (2151KB)

http://www.portalmedico.org.br/include/biblioteca virtual/medico e seu trabalho/regiao nordeste/nordeste final.PDF

Carneiro MB, Gouveia VV, Souza E (coords). 0 médico e seu trabalho: resultados da região Sudeste e seus estados. Brasília, Conselho Federal de Medicina, 2005. 188 p. (1470KB)

http://www.portalmedico.org.br/include/biblioteca virtual/medico e seu trabalho/regiao sudeste/Sudeste.PDF

Hunt R. et al. (eds.) Microbiology, Virology, Immunology, Bacteriology, Parasitology, Mycology Online. Columbia, Department of Pathology, Microbiology and Immunology, 2006. (on line)

http://pathmicro.med.sc.edu/book/welcome.htm

MEDICINA E SOCIEDADE

Pereira, JCM. Medicina, saúde e sociedade. Ribeirão Preto, Complexo Gráfico Villimpress, 2003. 219p. (718KB)

http://www.fmrp.usp.br/rms/publicacao/medicina\%20saude\%20sociedade\%20alterado.pdf

\section{MEDICINA - PROCEDIMENTOS}

Paiva EV, Giron AM. Classificação brasileira hierarquizada de procedimentos médicos. 4.ed. São Paulo, Associação Médica Brasileira, 2005. 199p. (771KB)

ISBN: 85-89073-03-3

http://www.ramb.org.br/CBHPM 4 Edicao.pdf

\section{NEUROESQUISTOSSOMOSE}

Guia de vigilância epidemiológica e controle da mielorradiculopatia esquistossomótica. Brasília, Ministério da Saúde, Secretaria de Vigilância em Saúde, 2006. 32 p. (Série A Normas e Manuais Técnicos) (566KB)

ISBN 85-334-1260-6

http://portal.saude.gov.br/portal/arquivos/pdf/guia mielo esquisto.pdf

PRIMEIROS SOCORROS

Oliveira Cardoso TA0. Manual de primeiros socorros. Brasília, Ministério da Saúde; Rio de Janeiro, Fundação Oswaldo Cruz, 2003. 207p. (8.99MB)

http://www.dominiopublico.gov.br/pesquisa/DetalheObraForm.do?select_action=\&co_obra=19486

http://www.dominiopublico.gov.br/pesquisa/Detalhe0braDownload.do?select action=\&co obra $=19486 \& c 0 \quad$ midia $=2$ 


\section{ROEDORES}

Manual de controle de roedores. Brasília, Ministério da Saúde, Fundação Nacional de Saúde, 2002. 132p. (5.2MB)

http://portal.saude.gov.br/portal/arquivos/pdf/manual roedores.pdf

\section{SAÚDE PÚBLICA}

100 anos de Saúde Pública: a visão da Funasa. Brasília, Fundação Nacional de Saúde, 2004. 234 p. (11197KB)

http://www.funasa.gov.br/Web\%20Funasa/pub/pdf/livro 100-anos.pdf

Diretrizes para implantação do Projeto Saúde e Prevenção nas Escolas. Brasília, Ministério da Saúde. Secretaria de Vigilância em Saúde, Programa Nacional de DST e Aids, 2006. 24p. (Série Normas e Manuais Técnicos, No. 77) (152KB)

ISBN 85-3334-1256-88

http://www.aids.gov.br/services/DocumentManagement/FileDownload.EZTSvc.asp?DocumentID=\{2D43C72E-C36F-445A21C1-C35091DD9E60 $\}$ \&ServiceInstUID $=\{$ B8EF5DAF-23AE-4891-AD36-1903553A3174 $\}$

Duarte, EC et al. Epidemiologia das desigualdades em saúde no Brasil: um estudo. Brasília, Organização Pan-Americana da Saúde, 2002. 123 p. (590KB)

ISBN $85-87943-09-\mathrm{X}$

http://portal.saude.gov.br/portal/arquivos/pdf/epi desigualdades.pdf

Educação profissional básica para agentes indígenas de saúde: módulo promovendo a saúde e prevenindo parasitoses intestinais e doenças de pele. Brasília, Fundação Nacional de Saúde, 2005. 38 p. (848KB)

http://www.funasa.gov.br/Web\%20Funasa/pub/pdf/Parasitoses.pdf

Especial cidadania: dicas de saúde publicadas no Jornal do Senado. Brasília, Senado Federal, 2005; 72p.

http://www.senado.gov.br/jornal/arquivos jornal/arquivosPdf/LivroCidadaniaSaude.pdf

Guia para a formação de profissionais de saúde e de educação. Saúde e prevenção nas escolas. Brasília, Ministério da Saúde, 2006. 180p. (Série Manuais No. 75) (887KB)

http://www.aids.gov.br/services/DocumentManagement/FileDownload.EZTSvc.asp?DocumentID=\{C969F2FC-D056-4AB18B49-558AD6D44DA2 $\} \&$ ServiceInstUID $=\{$ B8EF5DAF-23AE-4891-AD36-1903553A3174 $\}$

National Center for Health Statistics Health, United States, 2005 with chartbook on trends in the health of Americans. Hyattsville, Centers for Disease Control and Prevention, 2005. 550p. (DHHS Publication No. 2005-1232) (10238KB)

http://www.ncbi.nlm.nih.gov/books/bookres.fcgi/healthus05/healthus05.pdf

www.cdc.gov/nchs/hus.htm.

National Center for Health Statistics Health, United States, 2006 with chartbook on trends in the health of Americans. Hyattsville, Centers for Disease Control and Prevention, 2006. 559p. (DHHS Publication No. 2006-1232) (8021KB)

http://www.cdc.gov/nchs/data/hus/hus06.pdf

http://www.cdc.gov/nchs/hus.htm

Saúde Brasil 2006: uma análise da situação de saúde no Brasil. Brasília, Ministério da Saúde, Secretaria de Vigilância em Saúde, Departamento de Análise e Situação em Saúde, 2006. 620p. (Série G Estatística informação em Saúde) (19.3MB)

ISBN 5-334-1223-11

http://portal.saude.gov.br/portal/arquivos/pdf/saude brasil 2006.pdf 
Saúde da população negra no Brasil: contribuições para a promoção da eqüidade. Brasília, Fundação Nacional de Saúde, 2005. 446p. $(9.81 \mathrm{~KB})$

http://www.funasa.gov.br/Web\%20Funasa/pub/pdf/popneg.pdf

A saúde da população negra e o SUS: ações afirmativas para avançar na eqüidade. Brasília, Ministério da Saúde, Secretaria-Executiva, Subsecretaria de Planejamento e Orçamento, 2005. 60 p. (Série BTextos Básicos em Saúde) (659KB)

ISBN 85-334-0889-7

http://dtr2001.saude.gov.br/editora/produtos/livros/pdf/05 0630 M.pdf

\section{SÍFILIS}

Diretrizes para o controle da sífilis congênita: Manual de bolso. 2.ed. Brasília, Ministério da Saúde, Secretaria de Vigilância em Saúde, Programa Nacional de DST/Aids, 2006. 73p. (Coleção DST/Aids Série Manual, No. 24) (662KB)

ISBN 85-334-1157-X

http://www.aids.gov.br/services/DocumentManagement/FileDownload.EZTSvc.asp?DocumentID=\{275F9E73-FD58-41AD809B-E8B0A81EB242 $\}$ \&ServiceInstUID $=\{$ B8EF5DAF-23AE-4891-AD36-1903553A3174 $\}$

Curso básico de vigilância epidemiológica em sífilis congênita, sífilis em gestante, infecção pelo HIV em gestantes e crianças expostas. Brasília, Ministério da Saúde, Secretaria de Vigilância em Saúde, 2006. 216 p. (Série A. Normas e Manuais Técnicos, No. 78) $(1.475 \mathrm{~KB})$

ISBN 5-334-1264-99

http://www.aids.gov.br/services/DocumentManagement/FileDownload.EZTSvc.asp?DocumentID=\{BB5F4053-F5A3-4A0DA38B-770310ED32A7 $\}$ \&ServiceInstUID $=\{$ B8EF5DAF-23AE-4891-AD36-1903553A3174 $\}$

\section{TRABALHOS CIENTÍfICOS}

Kamps BS Free Medical Information: Doctor = Publisher. Paris, Flying Publisher, 2005. 100 p. [* Erro interno: Formato de arquivo inválido. I incorporado.WMF *1 (1.2MB)

ISBN 3-924774-47-1

http://www.freemedicalinformation.com/download2.htm

http://freemedicalinformation.com/freemedicalinformation.pdf

\section{TRANSFUSÃO DE SANGUE}

Manual técnico de hemovigilância. Brasília, Ministério da Saúde, Agência Nacional de Vigilância Sanitária, 2004. 40p. (Série A Normas e Manuais Técnicos) (787KB)

http://dtr2001.saude.gov.br/editora/produtos/livros/pdf/04 0700 M\%20.pdf

Manual técnico para investigação da transmissão de doenças pelo sangue. Brasília, Ministério da Saúde, Agência Nacional de Vigilância Sanitária, 2004. 110p. (Série A Normas e Manuais Técnicos) (1.7MB)

ISBN 85-334-0810-2

http://www.aids.gov.br/services/DocumentManagement/FileDownload.EZTSvc.asp?DocumentID=\{6B22B533-71C5-44289A85-BC92677A5F29 $\} \&$ ServiceInstUID $=\{$ B8EF5DAF-23AE-4891-AD36-1903553A3174 $\}$

\section{VACINAS}

Vigilância dos eventos adversos pós-vacinação: cartilha para trabalhadores de sala de vacinação. Brasília, Ministério da Saúde, Secretaria de Vigilância em Saúde, Departamento de Vigilância Epidemiológica, 2003. 147p. (Série F Comunicação e Educação em Saúde) (9.22MB)

ISBN 85-334-0759-9

http://portal.saude.gov.br/portal/arquivos/pdf/cartilha eadv nivel medio.pdf 


\section{VIGILÂNCIA EPIDEMIOLÓGICA}

Guia de vigilância epidemiológica. Volume I - Aids / Hepatites Virais. Brasília, Ministério da Saúde, 2002. 447p. (2436KB)

http://bvsms.saude.gov.br/bvs/publicacoes/funasa/guia_vig_epi_vol_l.pdf

Guia de vigilância epidemiológica. Volume 2 - Influenza / Varíola. Brasília, Ministério da Saúde, 2002. 399p. (2163KB)

http://bvsms.saude.gov.br/bvs/publicacoes/funasa/guia vig epi vol ll.pdf

Guia de vigilância epidemiológica. 6.ed. Brasília, Ministério da Saúde. Secretaria de Vigilância em Saúde, 2005. 816p. (Série A Normas e Manuais Técnicos) (5120KB)

ISBN 85-334-1047-6

http://portal.saude.gov.br/portal/arquivos/pdf/Guia_Vig_Epid_novo2.pdf

\section{ENDEREÇOS REDIRECIONADOS REFERENTES AO ARTIGO PUBLICADO NA REVISTA DA SOCIEDADE BRASILEIRA DE MEDICINA TROPICAL 36: 113-127, 2003.}

\section{Animais peçonhentos}

Manual de diagnóstico e tratamento de acidentes por animais peçonhentos. 2.ed. rev. Brasília, Ministério da Saúde; Fundação Nacional da Saúde, 2001. 112p. (2.3MB)

http://portal.saude.gov.br/portal/arquivos/pdf/manu_peconhentos.pdf

\section{Biossegurança}

Rickmond, Jonathan Y. \& McKinney, Robert W., ed. - Biossegurança em laboratórios biomédicos e de microbiologia;. Brasília, Ministério da Sáude; Fundação Nacional de Saúde, 2001. 291p. (9968KB)

http://portal.saude.gov.br/portal/arquivos/pdf/Livro Biosseguranca.pdf

\section{Dengue}

Dengue instruções para pessoal de combate ao vetor: manual de normas técnicas. 3.ed. rev. Brasília, Ministério da Saúde; Fundação Nacional de Saúde, 2001. 75p. (1425KB)

http://portal.saude.gov.br/portal/arquivos/pdf/ipcv 001.pdf http://portal.saude.gov.br/portal/arquivos/pdf/ipcv 002.pdf http://portal.saude.gov.br/portal/arquivos/pdf/ipcv 003.pdf http://portal.saude.gov.br/portal/arquivos/pdf/ipcv 004.pdf http://portal.saude.gov.br/portal/arquivos/pdf/ipcv 005.pdf http://portal.saude.gov.br/portal/arquivos/pdf/ipcv 006.pdf http://portal.saude.gov.br/portal/arquivos/pdf/ipcv 007.pdf http://portal.saude.gov.br/portal/arquivos/pdf/ipcv_008.pdf http://portal.saude.gov.br/portal/arquivos/pdf/ipcv 009.pdf http://portal.saude.gov.br/portal/arquivos/pdf/ipcv 010.pdf http://portal.saude.gov.br/portal/arquivos/pdf/ipcv_011.pdf http://portal.saude.gov.br/portal/arquivos/pdf/ipcv 012.pdf http://portal.saude.gov.br/portal/arquivos/pdf/ipcv 013.pdf http://portal.saude.gov.br/portal/arquivos/pdf/ipcv 014.pdf http://portal.saude.gov.br/portal/arquivos/pdf/ipcv 015.pdf http://portal.saude.gov.br/portal/arquivos/pdf/ipcv 016.pdf 


\section{Doenças Infecciosas e Parasitárias}

Doenças infecciosas e parasitárias: aspectos clínicos, de vigilância epidemiológica e de controle. Guia de bolso. 2.ed.rev.ampl. Brasilia: Ministério da Saúde, Fundação Nacional de Saúde, 2000. 215 p. (947KB)

http://bvsms.saude.gov.br/bvs/publicacoes/funasa/GBDIP001 total.pdf

\section{Epidemiologia}

LIRA, Margarida M.T. de Azevedo \& Drumong Júnior, Marcos - Estudos epidemiológicos. Brasília, Ministério da Saúde; Fundação Nacional de Saúde, 2000. 117p. (449KB)

http://portal.saude.gov.br/portal/arquivos/pdf/estudos epidemiologicos.pdf

\section{Febre amarela}

Manual de vigilância epidemiológica de febre amarela. Brasília, Ministério da Saúde; Fundação Nacional da Saúde, 1999. 59p. (575KB)

\section{http://portal.saude.gov.br/portal/arquivos/pdf/manu_feam.pdf}

\section{Imunização}

Comitê Técnico Assessor de Imunizações do Ministério da Saúde. Manual dos Centros de Referência de Imunobiológicos Especiais. Brasilia, Ministério da Saúde; Fundação Nacional da Saúde, 2001. 120p. (399KB)

http://portal.saude.gov.br/portal/arquivos/pdf/manual cries.pdf

Manual de normas de vacinação. 3.ed. Brasilia, Ministério da Saúde; Fundação Nacional de Saúde, 2001. 58p. (289KB)

http://portal.saude.gov.br/portal/arquivos/pdf/manu normas vac.pdf

Manual de vigilância epidemiológica dos eventos adversos pós-vacinação. Brasília, Ministério da Saúde; Fundação Nacional da Saúde, 1998. 102p. (515KB)

http://portal.saude.gov.br/portal/arquivos/pdf/manu eadpv.pdf

Recomendações para vacinação em pessoas infectadas pelo HIV. Brasília, Ministério da Saúde; Fundação Nacional de Saúde, 2002. 21p. (138KB)

http://portal.saude.gov.br/portal/arquivos/pdf/vacinacao hiv.pdf

\section{Leishmaniose Tegumentar Americana}

Manual de controle da Leishmaniose tegumentar americana. Brasília, Ministério da Saúde; Fundação Nacional de Saúde, 2000. 62p. $(1.2 \mathrm{MB})$

http://portal.saude.gov.br/portal/arquivos/pdf/manu leishman.pdf

\section{Malária}

Manual de terapêutica da malária. Brasília, Ministério da Saúde, Fundação Nacional de Saúde, 2001. 103p. (451KB)

http://portal.saude.gov.br/portal/arquivos/pdf/manu terapeutica malaria.pdf

\section{REFERÊNCIAS}

1. Gomes SP. Livros on-line da área médica. Revista da Sociedade Brasileira de Medicina Tropical, 36: 113-127, 2003.

2. Lopes IL. Novos paradigmas para avaliação da qualidade da informação em saúde recuperada na Web. Ciência da Informação, 31: 81-90, 2004. 